\title{
Participation des militaires à la sécurité intérieure : Royaume-Uni, Irlande du Nord
}

Didier Bigo, Emmanuel-Pierre Guittet et Andy SMITH

\section{(2) OpenEdition \\ 1 Journals}

Édition électronique

URL : http://journals.openedition.org/conflits/1608

DOI : 10.4000/conflits. 1608

ISSN : $1777-5345$

Éditeur :

CCLS - Centre d'études sur les conflits lilberté et sécurité, L'Harmattan

Édition imprimée

Date de publication : 1 décembre 2004

Pagination : 11-34

ISBN : 2-7475-7598-X

ISSN : 1157-996X

Référence électronique

Didier Bigo, Emmanuel-Pierre Guittet et Andy SMITH, « Participation des militaires à la sécurité intérieure : Royaume-Uni, Irlande du Nord », Cultures \& Conflits [En ligne], 56 | hiver 2004, mis en ligne le 28 avril 2005, consulté le 30 mars 2021. URL : http://journals.openedition.org/conflits/1608; DOI : https://doi.org/10.4000/conflits.1608

Ce document a été généré automatiquement le 30 mars 2021.

Creative Commons License 


\title{
Participation des militaires à la sécurité intérieure : Royaume-Uni, Irlande du Nord ${ }^{1}$
}

\author{
Didier Bigo, Emmanuel-Pierre Guittet et Andy SMITH
}

1 L'histoire de l'armée britannique, dans son rapport à la sécurité intérieure, peut, à bien des égards, se résumer à un double paradoxe. Le premier est celui d'une approche britannique officielle cherchant constamment à minimiser le rôle de l'armée dans la sécurité intérieure - et qui, lorsqu'il «faut » que l'armée y intervienne, insiste sur le fait que la doctrine appliquée est « pragmatique » - alors que se développent en Irlande du Nord une doctrine et des méthodes d'intervention qui rompent nettement avec ce modèle officiel. Le deuxième est celui d'une volonté de l'armée britannique de "vendre » à l'extérieur ce second modèle comme une réussite technique de haut niveau permettant une collaboration efficace entre toutes les forces de sécurité, alors que l'on assiste à la recherche discrète d'autres modèles, dont celui d'une troisième force de type police/armée et respectueuse du droit, qui réformerait les échecs bien réels du couple armée/police à l'anglaise.

2 L'organisation des forces de sécurité au Royaume-Uni a toujours fonctionné sur le plan théorique en termes de stricte séparation des fonctions et des organisations. Il n'existe pas, dit-on, de troisième force intermédiaire. Sur le plan juridique, il n'y a pas de collaboration civilo-militaire, au sens français ou italien du terme, pas plus qu'il n'y a de scénarios complexes, tenant compte de la gravité de la menace, pour un passage progressif de la prise de décision des civils vers les militaires avec une série de gardes fous établis à l'avance. Si l'armée intervient ce sera toujours de manière subordonnée dans le contexte très limité de la common law de l'aide aux pouvoirs civils. Seulement si ce discours officiel, minimisant le rôle de l'armée et son autonomie opérationnelle, tactique et stratégique, est pertinent en cas de catastrophe naturelle et d'aide aux pouvoirs civils, il n'a pas de prise sur la réalité lorsqu'on passe à la situation de l'Irlande. Ceci tient au fait que l'armée britannique, en l'absence de forces spécialisées dans le maintien de l'ordre public, a depuis des siècles été en première ligne lorsqu'il 
s'agissait de l'Irlande puis de l'Irlande du Nord. L'armée se sent impliquée, et surtout l'armée de terre. Pendant longtemps, la solution répressive a été la seule envisagée. La démocratisation, la montée des normes juridiques et de leur poids sur les décisions politiques et militaires ont changé, sinon la donne, du moins le discours. Depuis 1972, l'armée britannique a essayé différentes stratégies et a eu des relations de domination, de concurrence, de collaboration étroite et/ou de repli et de subordination à l'égard des forces de police. Elle a dû concilier selon les périodes, l'immobilisme du pouvoir politique de Londres, l'activisme des unionistes, les revendications des nationalistes, le tout sur fond de violence de la part des extrémistes. Son discours officiel a été rapidement institué comme un moyen de «maitriser ces variables » et non comme un discours de vérité. D'où l'importance que nous donnons, non pas au discours officiel luimême, mais à la manière dont, au-delà de celui-ci, une certaine doctrine de la sécurité intérieure a été traduite en actes dans le cas de l'Irlande du Nord. Dans cette perspective, il importe de rejeter un point de départ avancé dans le discours officiel qui postule que l'armée britannique a contribué à faire diminuer la violence politique dans ce territoire et à ramener l'Irlande du Nord à la normalité britannique. Non pas que l'armée n'ait pas œuvré pour la paix, mais au sens où ce discours est souvent anhistorique et se garde bien de revenir sur les périodes précédentes. L'armée n'a pas fait «qu'aider la police de l'Ulster à éradiquer le terrorisme ». Elle n'a pas été simplement « une force d'appui, derrière la Royal Ulster Company, supplétive et entièrement dans les mains du pouvoir civil ». La situation du code militaire pour l'ensemble des îles britanniques a pris un tour différent en Irlande du Nord de celui de la Grande-Bretagne et on ne peut pas comparer, sinon par ironie, les interventions des militaires en aide au pouvoir civil lors d'une tempête sur les côtes anglaises et leur rôle en Irlande du Nord. D'ailleurs, les lois spéciales ont organisé cette différence en donnant aux policiers, mais aussi aux militaires, des instruments spécifiques en matière de recherche, d'arrestation et de contrôle des foules. Il est donc plus vraisemblable comme hypothèse de départ de considérer qu'en Irlande du Nord, l'armée a fait partie des « institutions et des agences qui ont été conçues afin de gérer le conflit, mais qui en réalité ont renforcé et ont 'normalisé' l'idée que l'on peut simplement réduire ce conflit à la défaite du terrorisme $»^{2}$.

\section{Irlande du Nord et Grande-Bretagne : chez nous ou chez eux?}

3 On pourrait, en reprenant les analyses de Mathias Albert et Yosef Lapid sur les relations entre "identités, frontières et ordre $"^{3}$, dire que l'Irlande du Nord est un exemple particulièrement fort de l'avantage d'une analyse en termes de relations et de processus. Alors que la plupart des auteurs se sont concentrés sur la question des relations ordre et frontières, en mettant en avant les préoccupations de sécurité nationale, de protection du territoire, et parfois en réduisant ceci à la lutte antiterroriste, ce sont les deux autres axes du triangle qui expliquent le mieux les transformations sur le long terme des relations entre Irlande du Nord et GrandeBretagne. Le gouvernement britannique continue de structurer la représentation identitaire du Royaume-Uni à travers les quatre composantes que sont l'Angleterre, le Pays de Galle, l'Ecosse et l'Irlande du Nord. Dans la relation entre ordre et identité, le gouvernement a toujours été inquiet d'un effet domino interne où la séparation d'avec l'Irlande du Nord entraînerait une séparation avec l'Ecosse et même le Pays de Galle. 
Cela explique, pour beaucoup, une attitude qui insiste sur le fait que l'Irlande du Nord appartient au Royaume-Uni, qu'elle est un «chez nous». Attitude qui se concilie parfaitement avec les unionistes et, depuis peu, avec les nationalistes catholiques prêts à admettre un régime de simple autonomie renforcée. Seulement, la violence fréquente et intensive de l'Irlande du Nord et le climat créé par les lois d'exception ont lentement mais sûrement modifié la perception par les Anglais de leur rapport à l'Irlande du Nord. Alors que dans les années 1970, il ne faisait aucun doute pour une majorité d'Anglais que leur intervention était légitime car l'Irlande du Nord était chez eux, et qu'un vote avait sanctionné la partition, ils ont de plus en plus l'impression que l'Irlande du Nord est un " ailleurs ", un pays différent, marqué par la violence et le non respect du droit. Certains en viennent à penser en termes de pure inversion. Or, le conflit a moins changé en termes de modification des rapports de force entre les acteurs, de sécurité et d'ordre politique, qu'il n'a changé en termes de frontière et d'identité. Lorsque les acteurs anglais n'ont plus l'impression d'être chez eux en Irlande du Nord mais de soutenir des alliés, le sens du conflit change. En ce sens, le parallèle Irlande du Nord/ Kosovo et la mise en avant de discours au soutien de la paix d'une armée impartiale entre deux communautés autochtones ont "externalisé » l'Irlande du Nord. En parallèle, le rapport aux frontières a beaucoup joué. Les contrôles entre les deux îles du Royaume-Uni ont été permanents et la libre circulation interne n'a jamais véritablement existé depuis trente ans. Il est plus simple d'aller en Irlande qu'en Irlande du Nord, plus simple d'aller en France qu'en Irlande du Nord, du point de vue des contrôles à subir. Cela a joué par définition sur la dimension identitaire en distanciant les Anglais des Irlandais du Nord, mais aussi en rapprochant ces derniers, malgré leurs clivages, et en faisant de l'Irlande du Nord un pays à part, différent de l'Irlande de Dublin. Si l'Irlande du Nord avait été une colonie, le rapport au droit aurait été fondamentalement différent. En même temps le rapport politique aurait sans doute évolué plus tôt. Si l'Irlande du Nord avait été considérée comme exactement identique à la Grande-Bretagne, le gouvernement et la population n'auraient pas toléré la mise en place de lois spéciales, ne s'appliquant que sur une partie du territoire. En exceptionnalisant l'Irlande du Nord, par la multiplication de lois et de procédures spéciales, la dynamique de distanciation était enclenchée.

\section{Interaction entre processus violent et processus législatif d'exception}

4 En étudiant les aspects théoriques de la défense et de la sécurité au Royaume-Uni, il importe de dépasser un discours général sur ce sujet qui considère que ces forces de l'ordre sont « a-théoriques » et donc pragmatiques. Même si une doctrine sur ces sujets n'y est pas développée, débattue et enseignée aussi directement que dans certains autres pays, il n'en reste pas moins qu'un cadre normatif composé du droit et de règles professionnelles guide l'action de l'armée et de la police. Si, en termes généraux, ce cadre a peu évolué depuis de longues années, l'exemple du rôle des forces de l'ordre en Irlande du Nord montre, parfois jusqu'à la caricature, à la fois sa souplesse et ses conséquences voulues et non voulues. De manière générale, selon le discours officiel, le rôle traditionnel de l'armée britannique dans la sécurité intérieure s'est résumé à celui de «l'aide militaire au pouvoir civil ». Toutefois, derrière cette phrase à l'apparence bénigne se cache une longue histoire d'implications de soldats britanniques dans le 
maintien de l'ordre dans leur propre pays ${ }^{4}$. Dès l'arrivée en Irlande du Nord, la présence de l'armée dans les rues, à l'aéroport, tranche avec la situation anglaise, même lorsqu'il y a application des mesures de sûreté à la suite du 11 Septembre 2001. D'un côté des militaires armés de pistolet mitrailleur, de l'autre des policiers à grands tabliers jaunes. Il est clair que les investissements des militaires et leur visibilité sont différents d'une île à l'autre. Bien que l'armée britannique insiste sur le fait qu'elle agisse en Irlande du Nord comme elle agirait en tout autre point du territoire britannique si des actions terroristes s'y développaient, elle ne peut se départir d'un rapport particulier à l'île d'Irlande qu'elle a depuis son origine. En créant une armée de métier dès 1660 , les pouvoirs publics britanniques se sont progressivement donné un moyen, certes limité pendant de longues années, de mettre fin par la violence à des conflits internes, et l'Irlande a toujours été présentée comme l'exemple par excellence au moment de justifier l'existence d'une armée professionnelle. Ce rapport intime de l'armée à l'Irlande du Nord ne peut être masqué par le thème de l'aide au pouvoir civil. L'armée britannique se sent responsable de la situation irlandaise, et ce d'autant plus que le pouvoir politique britannique a toujours refusé jusqu'il y a peu, la création de forces spécialisées de type CRS ou de forces de police à statut militaire de type gendarmerie. Même si les officiers de l'armée ont souvent exprimé après la seconde Guerre Mondiale un malaise vis-à-vis de cette tâche "non-militaire » de "rétablissement de l'ordre», ils estiment que c'est leur devoir historique. Ils se trouvent donc dans "une position paradoxale " où l'armée constitue historiquement une composante essentielle du système de maintien de l'ordre et tient à y jouer un rôle, "alors que par ailleurs, elle demeure à beaucoup d'égards et est considérée comme une organisation extraordinaire, en particulier sur le plan de l'acceptabilité politique $»^{5}$. Elle a donc à subir une tension entre sa « respectabilité » et « son engagement » pour la sauvegarde de l'intégrité du territoire britannique.

5 On peut dire que son souci de respectabilité l'emporte de plus en plus et qu'elle voudrait, soit plus de liberté pour régler le conflit, soit un désengagement de plus en plus net, en laissant seules les forces de police gérer la question. Mais les hommes politiques n'ont voulu ni d'une solution, ni de l'autre. L'encadrement juridique par l'aide au pouvoir civil est alors un "pis-aller » qui ne satisfait véritablement personne, étant donné son cadre imprécis. Les civils y voient une sorte de blanc seing donné à l'armée. Les policiers quant à eux, sont certes ravis de pouvoir demander l'aide de l'armée, mais ils voudraient être maîtres aussi du temps où ils interviennent, et les militaires sont inquiets de devoir participer à des actions dont ils n'ont pas la maîtrise. Le principe de l'usage raisonnable de la force créé certes, à travers la jurisprudence qui l'accompagne, un guide d'action, renforcé par la technique des différentes fiches d'engagement selon les situations, mais on peut dire que l'essentiel repose sur les interprétations des lois spéciales à l'Irlande du Nord et non pas sur le principe général de l'aide au pouvoir civil mis en avant.

Une deuxième influence qui joue clairement sur la doctrine militaire britannique en matière de sécurité intérieure concerne son implication historique dans l'imposition, dans la consolidation et dans la gestion de l'Empire britannique. Plutôt que d'en faire un point général, il importe ici d'insister brièvement sur les expériences de l'armée britannique dans la décolonisation de plusieurs pays. Si les histoires globales de cette période tendent à souligner la nature non-violente de la fin de l'Empire britannique, il est plus exact de souligner un rôle non négligeable joué dans plusieurs cas par l'armée 
britannique. C'est en tout cas lors des tentatives de maitriser les « guérillas » rurales en Malaisie et au Kenya, ainsi que leurs homologues urbaines à Oman, que plusieurs officiers britanniques ont développé dans des approches et des méthodes de counter insurgency qui ont ensuite été intégrées comme des pratiques normalisées par l'armée britannique dans son ensemble ${ }^{6}$. Celles-ci ont souvent été ce qui de facto a inspiré les dirigeants de l'armée dans la conduite de leurs actions, et ce, au détriment d'une réflexion approfondie sur les rapports entre armée et police. Chaque institution a cherché à rester "maître » d'un domaine, plutôt que de chercher à institutionnaliser des rapports dans le cadre d'une collaboration civilo-militaire. Les rapports au pragmatisme et au localisme ont fait prévaloir des solutions ad hoc sur l'établissement de plans.

\section{La place extraordinaire de l'UDR : une soupape de sécurité ?}

7 Créé en vitesse en 1970 afin de remplacer les services spéciaux de la RUC (les B-specials), l'Ulster Defence Regiment (UDR) a constitué un élément central mais controversé du dispositif militaire en Irlande du Nord ${ }^{7}$. Renommé le Royal Irish Regiment (RIR) en 19928, ce régiment est mis sous le contrôle de l'armée régulière. En termes opérationnels, les officiers et les soldats de ce régiment, dont la majorité sont les réservistes travaillant à mi-temps, se sont concentrés essentiellement sur les activités de garde et de contrôle de voitures et de personnes. De fait, ils n'ont jamais été utilisés pour le maintien de l'ordre et le contrôle des émeutes. Il n'en reste pas moins que le personnel de ce régiment a souvent participé à des opérations de plus grande envergure et, à travers son inscription locale ${ }^{9}$, à des opérations de renseignement ${ }^{10}$. Si, sur un plan purement fonctionnel, l'UDR a permis de mettre en œuvre les politiques militaires en Irlande du Nord, deux des traits de son personnel ont plutôt tendu à saper sa légitimité.

8 Le premier trait concerne la représentativité du personnel censé favoriser l'intégration sociale. Alors qu'à son origine $10 \%$ de celui-ci est catholique, très vite ce chiffre se trouve en chute libre pour plafonner à $2 \%$ (dont beaucoup ne sont pas des catholiques de l'Irlande du Nord). Si les menaces et les meurtres perpétués par l'IRA sur les membres de l'UDR expliquent en grande partie la composition déséquilibrée de l'UDR, des études montrent que la culture interne du régiment a également défavorisé l'adhésion des catholiques ${ }^{11}$. Le second trait de l'UDR qui a entaché sa légitimité est le lien établi entre certains membres de son personnel et les milices loyalistes. Au cours des années 1980, ce lien est rendu public dans un nombre croissant d'affaires portées devant la justice, notamment celle des «UDR four » en 1986, évolution qui provoque un rapport gouvernemental critique (The Stevens report) en 1989-90. Outre l'implication des membres de l'UDR dans le meurtre de nombreux catholiques, ce rapport met en cause l'implication de certains d'entre eux dans le vol et la transmission d'armes et de renseignements secrets aux milices loyalistes. Sur le fond, concernant l'institution, ce rapport met en cause à la fois la sélection des soldats de l'UDR ainsi que leur formation initiale et continue ${ }^{12}$. Ces mises en causes récurrentes nécessitent d'être interprétées à la lumière de « la raison d'être » de l'UDR. Au départ, l'idée qui orientait la création de l'UDR était celle de "la solution la moins mauvaise ». Le discours était le suivant: il vaut mieux avoir un certain nombre de membres réels ou potentiels des milices en uniforme et sous le contrôle de l'Armée régulière que de les laisser développer des 
milices secrètes. Force est de constater que cette idée ne semble pas avoir eu les effets escomptés. Il semble donc plus salutaire de considérer que l'existence de l'UDR a fait partie d'un compromis secret entre le gouvernement britannique et les élites protestantes, compromis qui a arrangé le premier, aussi pour des raisons financières ${ }^{13}$. Constamment critiqué par le gouvernement irlandais et par le SDLP, l'UDR a effectivement changé de nom en 1992. En juillet de cette année, l'Ulster Defence Regiment et le corps des "Royal Irish Rangers" sont regroupés pour former le "Royal Irish Regiment»(RIR). Composé de sept bataillons, le RIR peut aligner six d'entre eux directement en Irlande du Nord. Le septième étant à la disposition de toute opération internationale. Composé d'environ 5000 hommes, le RIR comprend à la fois des soldats à plein temps (environ 3000 ) et des soldats à mi-temps. L'impact du discours militaire en termes de Peace Support Operations est tout à fait notable. Mais il est moins évident que ce régiment ait réellement changé quant à sa fonction, ou sa disposition vis-à-vis de la population catholique. Mis à part le cas spécial de l'UDR, pour l'armée de terre professionnelle, l'Irlande du Nord est l'endroit où les troupes vont s'aguerrir et apprendre le métier. Mais justement ce métier a évolué. Durant la période coloniale et même ensuite les Irlandais sont des « ennemis » qu'il faut réduire et pacifier. Ce n'est qu'ensuite qu'ils deviennent des citoyens à calmer, à ramener à l'ordre et la raison, voire à protéger des activistes armés de part et d'autre. Seulement ce qui est vécu par les uns comme une "policiarisation» de leur métier est vu par d'autres comme une «militarisation" de la société ; militarisation qui ne passe pas tant par les militaires comme organisation que par la mise en place d'organismes agissant paramilitairement dans le contexte de lois spéciales.

\section{Histoire de l'intervention militaire et lois spéciales en Irlande du Nord : traditions militaire, coloniale et d'exception}

9 Etant donné que l'Irlande a été la première colonie britannique, ainsi que le premier territoire à chercher à quitter cet Empire, il n'est guère surprenant ni que les militaires britanniques y aient une longue histoire d'interventions, ni que ces militaires aient par moment cherché à y transposer des méthodes testées dans d'autres guerres d'indépendance. Afin de retracer l'évolution de la doctrine militaire en matière de sécurité intérieure en Irlande du Nord, il est utile d'en faire une rapide genèse. Loin de dresser un tableau complet de l'histoire de l'intervention de l'armée britannique en Irlande et en Irlande du Nord, il s'agit plutôt de souligner les évolutions de l'approche générale adoptée par les militaires dans ce(s) territoire(s) et notamment de retracer la manière dont les forces de l'ordre ont participé pleinement à faire évoluer des lois spéciales introduites au nom de "la lutte anti-terroriste », afin d'analyser au plus juste quel type d'interaction il existe entre ces lois, leur interprétation et les pratiques des forces de sécurité. Dans le contexte de cette recherche, l'apport d'une histoire longue du conflit entre des Irlandais et des Britanniques est double.

10 Premièrement, elle permet de souligner que l'implication des soldats britanniques a marqué de manière indélébile la mémoire collective des nationalistes irlandais. En composant une force d'occupation largement au-dessus de la loi, pendant plus de trois siècles, les militaires de la couronne sont devenus des figures et des symboles détestés. La lutte des Irlandais pour leur indépendance a profondément marqué ceux-ci car elle a 
été obtenue par un rapport de forces et non par un jeu de négociations. Profitant d'une conjoncture sécuritaire propice - la première Guerre Mondiale - leur mouvement de libération prend forme et passe à l'acte en Irlande. Sans rentrer dans le détail de la guerre civile qui s'en est suivi, il importe de souligner d'une part que l'armée britannique a forcément été impliquée dans ce conflit et, d'autre part, que des forces de réserve ont été mobilisées. En effet, renforcée par des soldats protestants ayant combattu les allemands, cette réserve (les Black and Tans) a rapidement gagné une réputation de brutalité qui a laissé beaucoup de traces par la suite. La partition de l'Irlande est apparue pour les nationalistes comme un défi à la démocratie, un peu comme si l'on avait accepté en 1958 le plan de Constantine où l'on séparait l'Algérie utile qui serait restée française de la totalité de l'Algérie. Cela a certes évité au Royaume-Uni le problème des "rapatriés ", mais les Unionistes qui avaient pour eux d'être là depuis plus de 300 ans sont restés sur place et ont du vivre dans l'insécurité en cohabitant avec leurs adversaires sur un même territoire. La sécurité intérieure de la nouvelle " province » de l'Irlande du Nord a été très largement laissée dans les mains d'une police (The Royal Ulster Constabulary) et de sa réserve (The B-specials) peu représentatives, et peu respectueuses, de sa population dans son ensemble. Cette police a été plus la police d'un régime et d'une minorité que celle des deux communautés vivant sur le territoire, et c'est bien d'ailleurs ce qui ressort maintenant des discussions sur la paix. Il existe un contentieux historique lourd à oublier. Cette situation est liée aux choix - ou aux non choix - des hommes politiques britanniques. Mais le symbole de ces choix reste la participation de l'armée au maintien de l'ordre au moment où le rapport de force s'inversait en faveur des nationalistes. Cela peut s'atténuer conjoncturellement, et parfois la police de la RUC prend la place de l'ennemi principal dans les discours nationalistes, mais il n'est guère possible pour un nationaliste de croire que l'armée britannique pourrait être un Tiers impartial.

11 Deuxièmement, en intégrant les leçons d'une histoire longue de ce conflit, on comprend mieux aussi comment les militaires britanniques ont pu parfois développer une représentation méprisante des Irlandais et une vision de leur propre rôle qui légitimait une méthode d'intervention qu'ils n'ont jamais cherché à appliquer en Grande-Bretagne elle-même. Comme nous l'avons déjà signalé, pour beaucoup de militaires, l'Irlande du Nord n'est pas un « chez nous » mais une terre étrangère inclue dans le territoire britannique, et qui doit y rester pour des questions de géopolitique. Le paradoxe tient à ce que cette distanciation résulte des lois spéciales visant à " protéger l'identité du Royaume-Uni ». C'est depuis 1922 que les lois spéciales ont visé à maintenir coûte que coûte un territoire nord-irlandais. Elles ont peu à peu créé un climat d'exception permanente qui a construit un clivage identitaire interne au Royaume-Uni où le fait d'être protestant et d'avoir des liens historiques n'empêche pas les Anglais de considérer que les Irlandais du Nord, même protestants, ne leur ressemblent pas.

\section{Mise en retrait, « normalité » et retour à l'exception : 1940-1969}

12 En Grande-Bretagne et ailleurs, la représentation dominante des années entre la partition de l'Irlande, la fin de la deuxième Guerre Mondiale et la naissance d'un mouvement «des droits civiques» est celle d'une période relativement paisible. En 
effet, la violence par attentat est faible durant ces années si on la compare aux années d'avant ou d'après. Mais cette absence de violence est-elle liée à un plus grand bien-être remis en cause par la PIRA ou est-elle due à une hégémonie protestante remise en cause par la montée des droits civiques? Les interprétations diffèrent. Pour les protestants, l'Irlande du Nord avait un avenir prometteur remis en cause par les terroristes. En revanche, pour la minorité catholique nationaliste, on estime que cette période est la pire, avec la mise en place d'un dispositif sécuritaire partial agissant essentiellement au service des élites politiques et économiques protestantes. Reposant sur la RUC et les Bspecials, c'est ce dispositif qui en 1969 réagit très violemment aux manifestations organisées par le mouvement des « droits civiques » à Derry et à Belfast, qui encourage, voire qui participe à la mise à sac de quartiers catholiques par des «loyalistes». Seulement à Derry comme à Belfast, la réaction nationaliste est bien plus importante que prévue et la police débordée finit par faire appel à l'armée britannique. Comme les militaires n'ont de cesse de le rappeler, au départ, ils ont rencontré un accueil plutôt positif de la part de la population catholique parce qu'ils étaient perçus comme leurs protecteurs face aux "extrémistes " protestants mais aussi face à la police et à sa réserve. A cet égard, un des premiers actes de l'armée en 1969 a été le désarmement de la RUC et la suppression des B-specials. Mais, il lui est néanmoins impossible d'agir en Tiers impartial. Trop de liens avec les protestants se sont construits à différents niveaux. Il va donc y avoir rapidement un hiatus entre le discours officiel s'appuyant sur un recours momentané à l'armée face à une situation d'exception et les pratiques de la lutte antisubversive à quelques encablures de chez soi. L'armée hésite entre le modèle de la contre insurrection et celui du droit interne qui ne lui donne guère d'opportunité d'agir. Les hommes politiques quant à eux réactivent des lois spéciales concernant les armes à feu (1969 et 1971 firearms acts), la protection des personnes (1970), la détention d'explosifs (1970). Autant de lois où l'on a inversion de la charge de la preuve par rapport au système anglais. S'y ajoute l'interdiction des sit ins et la pénalisation des formes de désobéissance civile (1970, Criminal justice temporary provisions). A ce titre, la reprise de la violence en Irlande du Nord en 1969 offre un premier exemple de la manière dont le " partenariat » entre le Home Office, le Ministry of Defence (MOD) et l'armée n'a pas fonctionné selon les règles officielles.

13 Le premier élément qui a «brouillé » ce jeu d'acteurs a été l'existence d'un parlement et d'un gouvernement de l'Irlande du Nord, Stormont ${ }^{14}$. Un gouvernement au sein duquel certains élus protestants ne voulaient pas l'intervention de l'armée demandée, selon eux, par leur police, parce qu'ils craignaient que celle-ci ne mette en cause l'existence même de leur autonomie gouvernementale. Et l'avenir ne leur donna pas tort. Par conséquent, pendant près de trois ans le contrôle politique de l'armée se trouva divisé entre les élus de Westminster et ceux de Stormont, d'une part, et entre le MOD et le Home Office, d'autre part. L'armée, paralysée sur le plan stratégique par les orientations contradictoires, proteste mais maintient un haut niveau de légalisme. Cela explique le silence que s'impose la hiérarchie militaire. Il ne faut pas donner l'impression de critiquer le gouvernement. C'est à ce moment là que commence le premier paradoxe que nous signalons en introduction. L'armée, obéissant au pouvoir politique de Londres, s'en tient au discours officiel d'une aide aux pouvoirs civils dès cette époque mais développe une doctrine d'emploi des forces qui s'apparente à la lutte antisubversive et à l'expérience de la décolonisation. 


\section{Stormont et la RUC, Westminster et l'armée. Un jeu à quatre : 1969-1972}

14 Avec la suppression des B-specials, Stormont semble perdre le contrôle. Quant à l'Ulster Defense Regiment il fait désormais partie intégrante de l'armée britannique. L'armée pense qu'elle va pouvoir agir comme elle l'entend et croit à l'époque pouvoir régler rapidement la situation. A cette occasion les troupes des SAS («Special Air Services») patrouillent dans les rues de Belfast. Ce corps militaire créé en 1941 par David Stirling obtient dès lors progressivement la nouvelle mission de contre-terrorisme en Irlande du Nord. Mais les SAS ne sont pas le seul corps militaire d'action contre-terroriste désormais présent sur le terrain. De nombreuses autres unités spéciales, plus ou moins reconnues publiquement, opèrent en Irlande du Nord contre la PIRA, point de focalisation des débats et des ressources policières et militaires. A cet égard, la 4th Field Survey Troop ( $4^{\text {th }} \mathrm{FST}$ ) a pour but de couvrir les missions d'infiltration des SAS et des soldats de l'armée britannique, formés par cette unité spéciale en Ulster. Elle était cantonnée au début, au sein d'un régiment du génie à Castle Dillon. Elle devient en 1970 "Intelligence and Security Group/Northern Ireland». Le gouvernement britannique n'a jamais reconnu l'existence de ces unités sur lesquelles porte le débat en matière d'intervention des militaires dans la "sale guerre ». Il existe aussi depuis 1974 à Belfast et à Londonderry la 14th Intelligence Company (14 $4^{\text {th }}$ Int Coy ou Det $\left.{ }^{15}\right)$. Cette unité s'est développée indépendamment de la $4^{\text {th }} \mathrm{FST}^{16}$, grâce aux SAS. Cette unité est spécialisée dans la recherche du renseignement en Irlande du Nord. Elle dépend du Director of Special Forces depuis 1987. Il en est de même pour les trois régiments SAS et le SBS. Elle est sous le contrôle de l'armée. Cependant, elle dispose de conseillers appartenant au MI5. Elle ne dispose que d'une cinquantaine d'hommes issus de l'armée britannique. Ils ont des missions de surveillance clandestine très longues. Ils sont formés par les SAS et le Joint Services Intelligence Training Center.

On voit donc l'ampleur des moyens que l'armée est prête à utiliser. Par ailleurs, l'Etat major a un plan qui suit dans les grandes lignes les recommandations de l'ouvrage du major Franck Kitson qui est publié en 1971, mais dont Kitson a donné des extraits importants dans les écoles de formation militaire dès le milieu des années 1960 . Ce ou ces plans reprennent l'articulation du livre. Il s'agit d'identifier dans un premier temps les techniques et tactiques de l'ennemi et de se rappeler comme Trinquier l'a signalé, que «le conflit se gagne par le soutien de la population $»^{17}$. Il faut donc tirer les leçons de la Malaisie et du Kenya en essayant au plus tôt de couper les racines du groupe violent en créant le doute au sein de la population qui le soutient sur ses intentions réelles. Il faut donc une politique de communication avec les nationalistes et les catholiques dans le cas britannique, insistant sur le fanatisme et le socialisme marxiste de la PIRA. Dans un deuxième temps, on infiltrera l'organisation pour l'éradiquer et on collaborera avec les administrations civiles pour légitimer les actions. Kitson, dans sa vision de la collaboration civilo-militaire, dit clairement que l'armée doit convaincre les civils de participer à la tâche de renseignement et que l'armée doit comprendre son rôle qui est d'impulser une stratégie acceptée par les autres forces au lieu de vouloir commander. Travaillant lui-même à Belfast, il cherche entre 1971 et 1974 à réorganiser l'armée afin de mieux identifier et de combattre les adversaires en responsabilisant chaque " company commander $»^{18}$. Chaque unité doit ficher chaque garçon de son secteur de plus de 12 ans et chaque fille de plus de 14 ans. En effet, dès 1973, Kitson introduit l'usage 
des ordinateurs, et plus de surveillance à long terme (les soldats étant formés souvent par les membres du SAS). Il critique ainsi les Etats-majors insensibles à la dimension nouvelle des guerres subversives. Seulement sa conception du civilo-militaire fait de l'armée, non pas une organisation subordonnée à la police, mais une organisation qui coordonne. Il lui semble dangereux de commencer des actions contre subversives tant que l'on ne sait pas qui centralise le renseignement et quelles sont les lois qui seront appliquées. Il commente négativement les pays qui ont des services cloisonnés de renseignement entre la police et l'armée, et prône l'idée d'action conjointe avec un bureau de liaison permanent à défaut d'organisation unique. En parallèle avec le renseignement, il faut une action psychologique contrant la propagande ennemie, et il propose l'usage de films, de reportages dans les journaux, visant à modifier l'état d'esprit de la population. Cependant, Brian Faulkner ne veut pas impliquer plus avant les Britanniques et fait accepter le 9 Août 1971 la politique des internements administratifs, contre l'avis de l'armée. La RUC est alors chargée en priorité de mener à bien cette politique.

Seulement celle-ci est faite sans discrimination et précipite les événements en poussant dans les bras des mouvements civiques nationalistes des centaines de personnes qui trouvent ces internements injustifiés. En Novembre 1971, le Compton Report évoque la grande brutalité des internements et les mauvais traitements. Il propose la dissolution de la RUC et la mise en place d'une police à l'anglaise, mais son rapport est considéré comme irréaliste, y compris à Londres ${ }^{19}$. Néanmoins, le 22 mars 1972 Edouard Heath, le Premier ministre britannique demande à ce que toutes les questions de sécurité soient transférées à Westminster car les protestations se multiplient. Faulkner démissionne le lendemain. Le 30 Mars 1972 est publié le Northern Ireland Act 1972 qui proroge le parlement de Stormont et permet à Westminster de faire la loi en Irlande du Nord. Le 31 mars 1972, le gouvernement britannique supprime le gouvernement de Stormont, impose un mode de gestion directe (direct rule) et crée son premier Secrétaire d'Etat pour l'Irlande du Nord ${ }^{20}$, ainsi qu'un Northern Ireland Office (NIO). Beaucoup d'analystes pensaient que l'administration directe par Westminster allait être le moyen de « normaliser » la situation. Le gouvernement britannique avait promis de suspendre les special acts, et il commença d'ailleurs par le faire.

C'est donc à partir de 1972 que l'armée britannique a dû travailler sous l'autorité du NIO à Belfast, en liaison avec le MOD et, plus ponctuellement, avec le Home Office. Plus exactement, ce dispositif de ministres et de haut fonctionnaires était censé encadrer les trois dimensions de l'implication de l'armée en Irlande du Nord: l'armée régulière, l'UDR (devenu plus tard le RIR) et les services spéciaux. Dans les premiers mois de l'administration directe, plus de 500 personnes furent relâchées. On sait maintenant que des contacts furent établis dès cette époque avec les leaders de la PIRA et du Sinn Fein (dont Gerry Adams, et Martin McGuiness), mais que ces contacts ne débouchèrent sur aucun compromis quant au statut futur de l'Irlande du Nord. La PIRA décida alors de reprendre une campagne d'attentats, alors que le gouvernement britannique était en train de réformer les techniques d'internement. On sait aussi que l'armée s'opposa à ces techniques d'internement mais que celles-ci furent maintenues sous pression de l'ex-gouvernement unioniste. 


\section{L'antiterrorisme en Irlande du Nord : pouvoirs accordés aux militaires et aux policiers}

18 A la suite de la campagne d'attentats de la PIRA en Juillet 1972, et du rapport Diplock, le nouveau secrétaire d'Etat à l'Irlande du Nord Roy Jenkins décida de réinstaurer les pouvoirs spéciaux. Trente ans après ils sont toujours en place, certes avec des modifications liées à l'activité jurisprudentielle des cours, mais sans doute en s'étant banalisés et étendus pour certains à l'ensemble du territoire britannique ${ }^{21}$. La commission Diplock cherche à régler la question des internements en trouvant d'autres alternatives « efficaces». Elle prévoit alors de ne plus faire la différence entre violence politique et crime. Elle établit une procédure qui garantit l'accès au juge. Mais en même temps elle propose la réforme de la procédure judiciaire, de la composition du tribunal et des modalités d'enfermement. Ses recommandations vont être suivies d'effets. Les pouvoirs de police et de l'armée dérivent pour beaucoup du 1973 Act remplaçant les Special powers précédents. Ainsi la section 10 du 1973 Act donne la possibilité aux membres de la RUC d'arrêter sans mandat toute personne qu'ils suspectent d'être terroriste. Un terroriste étant une personne qui commet ou qui a connaissance d'un acte ou de la préparation d'un acte de terrorisme, ou qui entraîne des personnes en vue de la commission de ces actes. Le terrorisme étant défini à son tour comme «l'usage de la violence en vue d'instiller la peur dans tout ou partie du public ». La personne tombant dans cette catégorie peut être détenue provisoirement durant 72 heures avant d'être inculpée et de pouvoir demander à voir un avocat. Les membres de la RUC se serviront dans les premières années de cette mesure pour interroger de très nombreuses personnes afin de leur soutirer des informations sur leurs proches et leurs voisins, tout en ne retenant pas de charges contre eux. C'est pourquoi les cours en 1987 exigèrent que les arrestations soient au moins fondées sur des suspicions raisonnables. Ce qui sera repris dans la section 18 du 1996 Northern Ireland Act. Ce dernier réduira à 48 heures la durée de la détention. Néanmoins la RUC peut toujours sur la base de la section $14 \mathrm{du}$ Temporary Provision Act on Prevention of Terrorism de 1989 qui reprend la section 14 du 1974 Act demander au secrétaire d'Etat à l'Irlande du Nord de prolonger la détention jusqu'à cinq jours. Mais ceci a été rarement utilisé. Concernant les soldats britanniques, ils jouissent d'un pouvoir spécial d'arrestation depuis la section $12 \mathrm{du} 1973 \mathrm{Act}$, reprise dans la section 19 du Northern Ireland Act de 1996. Cela permet à des soldats en activité d'arrêter sans mandat une personne s'ils estiment qu'elle a commis ou est sur le point de commettre une infraction. On les forme à ces pratiques avant leur arrivée en Irlande du Nord avec pour les soldats de base, des formations de deux mois soit dans le Sud-est de l'Angleterre (Lydd), soit en Allemagne. Organisées par le Northern Ireland Training Advisory Team (NITAT), ces formations de cinq jours portent sur la collaboration avec la police, les tactiques de leurs adversaires et les enjeux du conflit, avec ensuite un stage pratique (à Rype). Depuis 1987, comme pour les policiers, il leur faut maintenant montrer qu'ils avaient des suspicions raisonnables pour arrêter les personnes. La personne peut alors être arrêtée et éventuellement détenue durant quatre heures avant d'être remise obligatoirement à la police.

19 Les militaires interrogés sur leurs pratiques insistent sur le fait que le commandement militaire essayait toujours d'associer un policier à un groupe de soldats afin que ce soit lui qui procède à l'arrestation. Ils évitent depuis le milieu des années 1980 d'utiliser ce pouvoir d'arrestation, sauf "circonstances exceptionnelles", où un acte se commet 
sous leurs yeux et qu'il n'y a pas de policiers à proximité. Ils considèrent que cela joue plus en matière de dissuasion de la petite délinquance qu'en matière de lutte contre le terrorisme. Policiers comme soldats ont aussi le pouvoir discrétionnaire de stopper, de questionner et de fouiller des personnes dans la rue sur la base du 1973 Act repris par les sections 20-6 et 25 du Northern Ireland Act de 1996. Ils peuvent lui demander son identité, son adresse et ce qu'elle sait d'une autre personne. Le refus de réponse tout comme une réponse incomplète par rapport aux connaissances de la personne, sont en eux-mêmes une infraction. Il n'y a pas besoin d'une suspicion raisonnable pour ces pouvoirs. Très choquant pour les juristes britanniques, ce pouvoir n'est pourtant guère plus que les contrôles d'identité en termes de police administrative en France depuis les lois Pasqua. Ces pouvoirs ont beaucoup été utilisés pour contrôler à nouveau les zones catholiques à forte implantation de la PIRA. Ils visaient à marquer la présence britannique et à la rappeler à tout moment. Mais, là aussi, l'armée depuis le milieu des années 1980 a changé de stratégie et préfère plus de discrétion. Elle ne veut pas aller au contact de la population.

Policiers et soldats ont aussi la possibilité d'entrer et de fouiller dans les maisons, sans mandat, s'ils cherchent des armes ou des moyens de communication. Les sections 17-24 et 26 du Northern Ireland Act de 1996 reprennent sur ce point le 1973 Act. Ils ont à justifier d'une suspicion légitime. Ces pouvoirs ont été utilisés pour "insécuriser " certaines familles suspectées d'être acquises à la PIRA. Les fouilles avaient lieu de manière très répétitive et sur tout un bloc d'immeubles. Là aussi les militaires ont préféré depuis plusieurs années ne plus participer à de telles opérations routinières, même s'ils estiment indispensable de conserver ces pouvoirs. Enfin, le secrétaire d'Etat pouvait prononcer une interdiction du territoire nord-irlandais à l'égard d'une personne suspectée de terrorisme, et avant 1995, n'était pas obligé de le lui signaler à l'avance. Mais après l'arrêt Gallagher de la Cour Européenne des Droits de l'Homme, il fallait la prévenir. Les critiques des juristes sur le droit à la vie familiale, à la propriété, à la libre circulation, ont amené le gouvernement à ne plus utiliser ce pouvoir. Ceci étant, ces pouvoirs permettant de stopper et de questionner les personnes ainsi que de les fouiller eux et leurs bagages, sont particulièrement systématiques aux ports qui relient l'Irlande du Nord et la Grande-Bretagne. Les officiers d'immigration et les douanes les partagent avec les policiers et les militaires. Ce contrôle de la libre circulation interne au Royaume-Uni a souvent été discuté mais jamais remis en cause. Il a néanmoins comme effet de renforcer la différence Nous/Eux au quotidien et joue semble-t-il comme facteur de dissuasion auprès des Nord-Irlandais d'aller faire leurs courses ou du tourisme en Grande-Bretagne.

\section{La recherche d'une " solution militaire », appliquer la doctrine Kitson ? : 1973-1976}

21 S'il faut se garder de trop se fier à l'image de l'intervention militaire initiale à la demande des policiers de la RUC et sans véritable discussion politique entre Stormont et Westminster, il est encore plus clair que la «lune de miel» connue par l'armée britannique avec les nationalistes en Irlande du Nord a duré très peu de temps, moins de trois ans. En remplaçant la police de la RUC liée à Stormont, en dépendant de Westminster à partir de 1973, l'armée a dû rapidement s'impliquer dans des opérations de divers types (maintien de l'ordre, arrestations, etc.) qui l'ont très vite transformée 
en cible « légitime » pour la PIRA. Il n'est pas certain que le commandement de l'armée voulait être engagé dans ces opérations qui remettaient en cause sa stratégie d'action psychologique visant à s'imposer comme un tiers et visant à déstabiliser la relation entre PIRA et nationalistes catholiques. Mais Londres ne donne pas de choix aux militaires et s'appuie sur ceux qui prônent, parmi les militaires, la voie de la lutte antisubversive. Ceux-ci doivent intervenir. Au même moment, la PIRA s'est recomposée et a su recruter parmi les membres des mouvements civiques. Dès 1971, la stratégie de la PIRA est de faire en sorte que l'armée devienne non seulement son ennemi, celui des nationalistes, mais aussi celui de toute la population catholique. L'engrenage commence. Chacun constitue l'autre en ennemi principal et cela va changer la dynamique du conflit.

22 En 1973, les militaires sont convaincus de pouvoir éradiquer la contestation rapidement. Ils voient la PIRA comme une organisation communiste révolutionnaire et font le parallèle avec les groupes de la RAF et des Brigades Rouges. Ils estiment que la PIRA vise à créer une révolution sur toute l'Irlande. Les documents de l'époque semblent impliquer que l'armée est en désaccord avec l'idée d'un simple maintien de l'ordre. Elle veut appliquer les pratiques de contre subversion : infiltration, jeu sur les médias, déstabilisation de la base nationaliste, mais avec des raisonnements simplistes qui feront que Kitson critiquera les bases de ces plans d'action en insistant sur le fait que l'Irlande du Nord est un cas à part. Kitson écrit en effet pour attirer l'attention de ceux qui se servent de ses idées en rappelant que la proximité géographique empêche la même relation au public que dans les conflits coloniaux. Il met l'accent sur la nécessité de convaincre l'opinion et de respecter plus de règles formelles. Il est un des premiers à proposer un retour vers la police en front line, quitte à la doter de moyens de coercition plus importants. D'autres responsables militaires analysent ce qu'ils appellent les lignes de fragilité de la communauté catholique et insistent sur le rejet de la violence par les femmes en proposant de favoriser leur expression. Ils mettent aussi en avant le rôle que les services de renseignement doivent jouer. Ils préconisent plus de sélectivité et "d'opérationalité ». Selon plusieurs témoignages, dès 1973-74, il circule des écrits insistant sur le fait qu'il ne faut pas se tromper d'ennemi, que celui-ci est la PIRA et les ultra-loyalistes qui usent de violence, non pas la population catholique des quartiers populaires. On abandonne le discours sur la guerre subversive au profit d'un discours sur le terrorisme. Même si les militaires agissent souvent de concert avec la RUC, ils n'hésitent pas, par moment, à agir seuls et à utiliser les quatre heures de délais qu'ils ont avant de remettre un suspect à la police. Seulement l'Etat major se divise sur cette politique où les militaires se substituent de plus en plus aux policiers et deviennent par voie de conséquence les premières cibles de la PIRA. Une partie des militaires refusent d'entrer dans une militarisation de l'action à l'égard de l'ensemble de la population catholique. Ils mettent en cause l'idée de zonage et insistent au contraire sur la nécessaire « individualisation » de l'action. Il leur semble plus utile de dissocier la PIRA des groupes catholiques que de mettre tous ces derniers sous surveillance. Dans ce cadre, diverses sources s'accordent pour dire que le commandement de l'armée n'a pas demandé la politique de "l'internement» des terroristes soupçonnés mise en place à partir de 1973, car cela allait à l'encontre de la politique globale de communication visant à montrer l'armée sous un jour favorable. Néanmoins les hommes politiques en décident autrement et il est certain que cette politique a participé à les transformer en adversaires résolus des catholiques, assimilés à la PIRA et au communisme. A court terme, la politique de contre subversion militaire 
et les techniques d'incarcération via les Cours Diplock aboutissent à beaucoup d'arrestations et à une réduction dans le nombre d'attaques à la bombe, de fusillades et d'émeutes. Mais cette "réussite» a été obtenue au prix d'un nombre important de morts et de blessés (tant chez les « terroristes » que chez les militaires) et, surtout, au prix d'un approfondissement de la séparation entre les représentants de l'Etat en Irlande du Nord et la population de la minorité catholique. Les hauts responsables militaires sont les premiers à s'en rendre compte et à reconnaître l'échec de la lutte contre subversive généralisée, mais ils se taisent en public.

\section{«Ulsterisation » et repli des interventions militaires : 1976-1998}

En partie à cause de ses pertes d'hommes, en partie à cause d'une évolution connue par la RUC, à partir de 1976, le discours officiel de l'armée sur son propre rôle en Irlande du Nord se modifie. Désormais, il s'agit de rendre à la police le rôle de leader en matière de sécurité intérieure à travers un processus surnommé « Ulsterisation ». En renforçant le dispositif policier, la mission de l'armée est devenue de «soutenir la RUC dans la défaite du terrorisme ». Dans les faits, ce changement de discours n'a pas forcément rapproché les militaires des policiers. Pendant de longues années les deux organisations se sont souvent comportées de manière autonome, voire contradictoire, notamment en matière de renseignement. Il n'en reste pas moins que l'Ulsterisation a progressivement libéré l'armée du rôle ingrat et dangereux du maintien de l'ordre, lui offrant ainsi l'occasion de se concentrer davantage sur la surveillance et sur les opérations secrètes. Au cours des années 1980, ce changement de stratégie a même débouché sur une autonomie très grande donnée aux services spéciaux de l'armée. Une autonomie qui s'est traduite, d'une part, par de nombreux assassinats de personnes soupçonnées de faire partie de la PIRA et, d'autre part, par des exemples de collusion entre ces services et des milices loyalistes. En effet, depuis l'accord anglo-irlandais de 1984, plusieurs groupes armés loyalistes ont relancé des campagnes de violence contre la population catholique de l'Irlande du Nord. Or, pendant de longues années ni la RUC, ni l'armée n'ont cherché à poursuivre ces groupes avec la même ardeur que celle accordé à l'IRA. L'armée semble néanmoins avoir joué un rôle de "modérateur ", au moins au niveau du MOD, dans le conflit. On ne peut savoir jusqu'où l'armée est impliquée dans la réussite des accords du Good Friday et si elle a accéléré le processus ou au contraire l'a freiné. Toujours est-il que durant les dix ans qui précédent cet Accord, l'armée a changé son discours sur l'Irlande du Nord. Il s'agit d'arriver à « une solution intercommunautaire» et la vision du conflit devient clairement celle d'un conflit intercommunautaire et non plus celle d'un conflit idéologique. Dans ce cadre, une compétition entre experts internes à l'armée se met en place et finalement, l'armée conceptualise son rôle et développe une stratégie de communication comme celui d'une force de peace enforcement « impartiale » et non plus comme une agence luttant contre la subversion. Dès lors, prendre contact avec des leaders nationalistes est «naturel ». Il s'agit d'entendre les plaintes de toutes les parties. De ce point de vue ne peut-on pas parler d'une «kosovisation» de l'Irlande du Nord? La doctrine des Opérations de Soutien de la Paix modifie la conception en terme de sécurité intérieure sous deux rapports. On traite de moins en moins l'Irlande du Nord comme un cas interne et l'aller-retour théorique entre Irlande et Kosovo, qui visait initialement à promouvoir 
une solution à l'anglaise au Kosovo face aux modalités à la française, ou la préférence pour un modèle dual de forces entre armée et police civile plutôt que le modèle triphasé armée, forces de gendarmerie, police civile, s'inverse. L'Irlande du Nord servait à lire le Kosovo. C'est désormais le Kosovo qui permet de lire l'Irlande du Nord. L'Irlande du Nord s'ethnicise et se désidéologise. L'armée y est en situation de "(re)construction de l'Etat» (nord-irlandais) tout autant qu'en opération interne. Il s'agit de "réconcilier les communautés locales antagonistes». On applique alors les « techniques de négociation » qui ont réussi plus ou moins en Afrique du Sud, en Bosnie et qui sont prônées pour le Kosovo à l'Irlande du Nord. Les comparaisons avec l'externe colonial qui était jugé inacceptable par le pouvoir politique redeviennent légitimes si, en contrepartie, l'armée accepte un discours général de "policiarisation » de ses tâches dans les opérations de maintien de la paix. Une lecture du Good Friday plus détaillée - et surtout des travaux préparatoires - permettrait sans doute d'étayer ce raisonnement. Néanmoins, plusieurs entretiens ont mis en avant ce rôle positif de l'armée dans la réconciliation. Mais qui n’a pas intérêt désormais à se dire initiateur des accords?

\section{La fin d'une intervention militaire de trente ans? « The Good Friday Agreement ": 1998 et ses suites}

Cet accord signé entre le gouvernement britannique et le gouvernement irlandais a été approuvé par presque $70 \%$ de la population irlandaise en mai 1998. Cet accord entérine la partition, en soumettant l'avenir de la province au vote de la majorité de ses électeurs ${ }^{22}$. L'Irlande du Nord demeure au sein du Royaume-Uni ${ }^{23}$, tant que la majorité le souhaitera, tout en prévoyant la mise en place d'institutions pan-irlandaises. L'Accord du Vendredi Saint (the Good Friday Agreement) propose la mise en place d'une Assemblée de 108 membres, qui exercera les pleins pouvoirs législatifs et exécutifs, sur la base d'un partage communautaire des fonctions. L'Autorité Exécutive, composée d'un Premier ministre et jusqu'à dix ministres, est élue par l'Assemblée, toujours selon le jeu de la parité communautaire. Ainsi, l'acceptation des lois se fait, à la fois, suivant la règle de la majorité qualifiée (60\%) ainsi qu'en présence d'au moins $40 \%$ de chacune des représentations communautaires, nationalistes et unionistes. Quant au Parlement britannique de Westminster, il reste en mesure de faire appliquer et respecter, en Irlande du Nord, toutes les lois relevant des obligations internationales du RoyaumeUni. Pour résoudre les problèmes de violence endémique en Irlande du Nord, cet accord repousse la solution pratique du conflit en espérant d'ici là un apaisement des tensions inter et intra communautaires. Pour cela, il affirme la nécessité d'un traitement non discriminatoire dans toutes les tâches politiques et quotidiennes pour les deux communautés. Et souligne que tout un chacun pourra choisir sa nationalité ou prendre la double nationalité ${ }^{24}$. L'accord précise que le traitement et la vérification de l'impartialité des statuts et fonctions entre les deux communautés sont gérés par une Commission de Paritée 25 . L'accord préconise aussi la mise en place d'un Conseil Ministériel entre l'Irlande du Nord et l'Irlande du Sud afin de développer consultations et coopérations, ainsi que la mise en place d'un Conseil anglo-irlandais (British-Irish Council, BIC), composé de représentants des gouvernements britannique et irlandais. Une des bases importantes de l'Accord, c'est la reconnaissance du statut des victimes de la violence comme nécessité pour la réconciliation ${ }^{26}$. A cet égard, une Commission des Victimes nord-irlandaises est mise en place. Mais, comme le souligne avec justesse Elise 
Féron, "le fait qu'un accord ait été conclu constitue certes une avancée considérable, mais la paix ne s'instaure pas par traité, surtout dans une société déchirée par des décennies de guerre civile larvée, cachée pudiquement derrière l'expression de 'troubles' «"27. Depuis la signature de l'accord de vendredi saint, en termes quantitatifs, la présence de l'armée britannique en Irlande du Nord a diminué. Le nombre de troupes en poste dans « la province » s'est réduit de manière considérable et, tout récemment, les manifestations les plus visibles de leur présence, tels que les "watchtowers", ont été démantelées. Pour autant, un nombre important de soldats reste en Irlande du Nord et on peut supposer qu'une partie du système de renseignement et de surveillance de l'armée demeure largement en place. Néanmoins, lorsque l'économie de la suspicion - qui va de pair avec la dévalorisation des droits civiques -, est relativisée, on peut espérer un rapprochement politique.

\section{NOTES}

1. Extrait du rapport La participation des militaires dans la sécurité intérieure: Royaume-Uni, Irlande du Nord, Centre d'études sur les conflits/DAS, 2001.

2. Ellison G., Smyth J., The Crowned Harp. Policing Northern Ireland, London, Pluto Press, 2000, p. ix.

3. Albert M., Jacobson D., Lapid Y. (Dir.), Identities, Borders, Orders. Rethinking International Relations Theory, University of Minnesota Press, 2001.

4. Mandeville A., "Armée et maintien de l'ordre au Royaume-Uni », Les Cahiers de la sécurité intérieure, n¹1-12, novembre 1992-avril 1993.

5. Ibid., p. 141.

6. L'ouvrage le plus connu est celui du major Franck Kitson, Low intensity operations. Subversion, Insurgency and Peacekeeping, Londres, Faber \& Faber, 1971. Dans la nouvelle préface à la réédition de 1991, l'auteur souligne sans ambages combien cet ouvrage a été rédigé pour faire face aux « troubles » de l'Irlande du Nord.

7. Ryder C., The Ulster Defence Regiment. An instrument of peace ?, London, Methuen, 1991.

8. Notons que de nombreux catholiques en Irlande du Nord ont contesté le nom de l'UDR en mettant en cause le terme de "Defence» (contre qui ?) et d'Ulster (nom utilisé par les protestants pour l'Irlande du Nord).

9. Du moins au début, l'UDR s'est organisé en sept bataillons, un par comté et un pour Belfast.

10. Ryder date cette implication de 1976. Ryder C., op. cit.

11. Ibid.

12. Entre 1970 et 1976 cette formation se réduit à six jours de cours. Après 1976, cette formation devient plus longue mais fait peu pour rapprocher les soldats de l'UDR à ceux de l'armée régulière.

13. Ryder estime qu'en 1989 l'UDR coûtait au gouvernement $£ 1$ million par semaine, alors que la RUC lui coûtait $£ 1.5$ millions par jour. Ryder, op. cit., p. 244.

14. Du nom de Stormont où siégèrent le Parlement et le gouvernement de la province.

15. Contraction de The Detachment.

16. Les deux unités (4th FST et 14th ISC) furent réunies au sein de l'Intelligence and Security Group/Northern Ireland. 
17. Voir Trinquier R., Guerre, subversion, révolution, Paris, Robert Lafond, 1968 ; Trinquier R., La guerre moderne, Paris, La Table ronde, 1961.

18. Hamill D., Pig in the middle. The Army in Northern Ireland 1969-1984,

Londres, Methuen, 1985, p. 121.

19. Trente ans seront perdus pour les soi-disant « réalistes ».

20. En la personne de William Whitelaw.

21. Cette importation vers la Grande-Bretagne des législations spéciales de l'Irlande du Nord est sans doute ce qui a été le moins étudié, mais ce qui ressort le plus des entretiens évoquant des peurs pour les libertés publiques. Voir plus loin l'article de Bigo D. et Guittet E.-P., «Vers une nord-irlandisation du monde?».

22. Good Friday Agreement, Constitutional Issues, (i) et (iii).

23. Ibid., annexe A, Draft Clauses/Schedules for incorporation in British legislation, $\$ 1$.

24. Ibid., Constitutional Issues, (vi).

25. Ibid., Strand One, Democratic Institutions in Northern Ireland - Safeguards, §5-(e): «An Equality Commission to monitor a statutory obligation to promote equality of opportunity in specified areas and parity of esteem between the two main communities, and to investigate individual complaints against public bodies ».

26. Ibid, Rights, Safeguards and Equality of Opportunity - Reconciliation and Victims of Violence, \$12: «The achievement of a peaceful and just society would be the true memorial to the victims of violence $»$.

27. Féron E., «Irlande du nord : une réconciliation incertaine», Cultures \& Conflits, $\mathrm{n}^{\circ} 40$, automne 1999, p. 16.

\section{RÉSUMÉS}

Cet article retrace l'histoire de l'intervention militaire britannique en Irlande puis en Irlande du Nord et vise à souligner les évolutions de l'approche générale adoptée par les militaires dans ce(s) territoire(s). L'armée, obéissant au pouvoir politique de Londres, s'en tient au discours officiel d'une aide aux pouvoirs civils mais développe en parallèle une doctrine d'emploi des forces qui s'apparente à la lutte antisubversive et à l'expérience de la décolonisation. En retraçant la manière dont les forces de l'ordre ont participé pleinement à faire évoluer des lois spéciales introduites au nom de la "lutte anti-terroriste", cet article apporte un éclairage sur les interactions qui existent entre ces lois spéciales et dérogatoires, leurs interprétations et les pratiques des forces de sécurité en poste en Irlande du Nord.

This contribution recounts the history of the British military intervention in Ireland and later in Northern Ireland, and aims at emphasizing the evolutions of the general approach adopted by the armed forces in this-these territory(ies). By obeying to London's political power, the army followed the official discourse of an aid to the civil power, but simultaneously developed a doctrine closer to the counter subversive fight and the decolonisation experiences. By underlining how the British armed forces have participated to the evolution of emergency laws to "fight against terrorism», this article sheds light on the interactions between these emergency and exceptional laws, their interpretation and the practices of the British armed forces in Northern Ireland. 
INDEX

Index géographique : Irlande du Nord

Index chronologique : 1970 - 1980, 1980 - 1990

Mots-clés : Doctrine militaire, sécurité intérieure, lutte anti-terroriste, militaires, antiterrorisme, contre-insurrection

Thèmes : British Army, Irish Republican Army (Provisional), Ulster Defence Regiment(UDF)

\section{AUTEURS}

\section{DIDIER BIGO}

Didier BIGO est Maître de Conférences des Universités à l'IEP de Paris. Chercheur associé au Centre d'Etudes et de Recherches Internationales (CERI) et Président du Centre d'Etudes sur les Conflits, il dirige les programmes cadres de recherche et développement européens ELISE (European Liberty and Security, PCRD5) et CHALLENGE (Changing the Landscape of European Security, PCRD6).

\section{EMMANUEL-PIERRE GUITTET}

Emmanuel-Pierre GUITTET est doctorant en sociologie politique au Laboratoire d'Analyses des Systèmes Politiques (LASP), Université Paris X-Nanterre, chargé de recherches au Centre d'Etudes sur les Conflits, il est aussi Junior Researcher dans les programmes ELISE et CHALLENGE (5ème et 6ème PCRD de la Commission européenne).

\section{ANDY SMITH}

Andy SMITH est directeur de recherche à la FNSP, directeur adjoint du Centre d'Etude et de Recherche sur la Vie Locale (unité mixte CNRS/FNSP). Il est spécialiste dans la sociologie des institutions européennes et des représentations de l'Europe. 